\title{
Populäre Wissenschaft: Metamorphosen des Wissens im Medium des Films
}

Jakob Tanner

\section{Summary}

Far from being merely a medium of simplification and conveyance of scientific facts, motion pictures exhibit an important epistemic function. On the one hand, the medium film is itself a product of research in various fields, on the other hand, it retroacts on perception and problem-solving in science, thereby influencing and changing research practices. The paper aims at describing these reciprocal effects and synergies by discussing two examples: first by the film "The principles of Einstein's theory of relativity", first released in Germany in 1922, second by the film "Mathematical image of the struggle for life", produced in 1937 for the inauguration of the "Palace of discoveries" in Paris, demonstrating the latest developments in evolutionary theory. It becomes evident that picture media have the capacity to transform the symbolic dimension of things and bodies, thereby offering new access to reality, which not only fascinated the spectators, but also inspired scientific research.

Keywords: science; film; popularisation; theory of relativity; biology

\section{Zusammenfassung}

Weit davon entfernt bloss ein Medium für die Vereinfachung und Vermittlung von wissenschaftlichen Erkenntnissen zu sein, kommt dem populären Film eine wichtige epistemische Funktion zu. Das Medium ist einerseits selber Produkt der Forschung in verschiedenen Bereichen, andererseits wirkt es auf die in der Wissenschaft dominierenden Wahrnehmungsweisen und

Jakob Tanner, Forschungsstelle für Sozial- und Wirtschaftsgeschichte der Universität Zürich, Rämistrasse 64, CH-8001 Zürich (jtanner@hist.uzh.ch). 
Fragestellungen zurück und verändert sie. Diese Wechselwirkungen und Synergien werden anhand zweier Beispiele erläutert: am 1922 in Deutschland uraufgeführten Film «Die Grundlagen der Einsteinschen Relativitätstheorie» und am 1937 gedrehten, zur Eröffnung des «Palais de la Découverte» in Paris gezeigten Film «Images mathématiques de la lutte pour la vie», der den neuesten Entwicklungsstand der Evolutionstheorie vorführte. Dabei zeigt es sich, dass die Bildmedien durch die symbolische Transposition von Dingen und Körpern neue Zugänge zur Wirklichkeit eröffnen, die nicht nur die Zuschauer faszinierten, sondern auch die Wissenschaft inspirierten.

Ein Kinobesuch hat gemäss einer weithin geteilten Erwartung vor allem gute Unterhaltung zu bieten. Schon in seinen Anfängen wurde er mit prickelndem Zeitvertreib und kommerziellem Vergnügen in Verbindung gebracht ${ }^{1}$. In der banalen Zerstreuung, die im Medium Film angelegt ist, könnte man einen Gegensatz zur Wissenschaft sehen, die sich in ihrem Selbstverständnis durch geistige Konzentration auszeichnet. «Wissenschaft im Film» und «Film in der Wissenschaft» wären dann störanfällige Konstellationen, weil hier zwei Bereiche aneinandergeraten, die sich schlecht vertragen. Eine solche Kontrastierung von Film und Wissenschaft würde allerdings rasch in einer analytischen Sackgasse enden. Weit aussichtsreicher ist es, von einem Komplementärverhältnis und synergetischen Beziehungen auszugehen. Die folgenden Ausführungen zeigen in einem ersten Kapitel einige allgemeine Zusammenhänge zwischen der visuellen Kultur des Kinos und wissenschaftlichen Forschungspraktiken auf und gehen im zweiten auf den Aufstieg des populären Wissenschaftsfilm ein. Im dritten Kapitel wird das Filmklischee des verrückten Wissenschaftlers und seiner Amok laufenden künstlichen Kreaturen dargestellt; diese Figuren haben das Bild der Wissenschaft im Film in der Zwischenkriegszeit massgeblich geprägt. Parallel zu dieser utopischen oder dystopischen, im Hauptzweck aber unterhaltenden Inszenierung der Wissenschaft im Spielfilm entwickelte sich ein neues Genre des wissenschaftlichen Popularisierungsfilms, das unter anderem konkrete (physikalische sowie biologische) Phänomene und abstrakte (mathematische) Zusammenhänge für ein interessiertes Publikum zugänglich zu machen versuchte. Kapitel vier und fünf behandeln exemplarisch zwei dieser Werke: den 1922 in Deutschland entstandenen und 1923 auch in einer englischen Kopie

1 Gunning 1990. 
vertriebenen Film über die Einstein'sche Relativitätstheorie und einen 1937 in Frankreich gedrehten Lehrfilm über die Darwin'sche Evolutionstheorie. Das abschliessende Kapitel fasst einige Ergebnisse des Aufsatzes zusammen.

\section{Synergien zwischen Wissenschaft und Film}

Obwohl Film und Wissenschaft nicht störungsfrei zusammenarbeiteten, überwogen dennoch die Synergien ${ }^{2}$. Diese stellen sich auf drei verschiedenen Ebenen ein: Erstens ist der Film das technische Medium eines verwissenschaftlichten Industriezeitalters. Er basiert auf science-based industries, er stellt eine Symbiose wissenschaftlicher Forschung und technischer Anwendungen dar ${ }^{3}$. Die verschiedenen materiellen Bestandteile, die im Kinemathographen vereinigt waren, von Kamera und Zelluloidstreifen über chemische Entwickler, Geräte für Schnitt sowie Montage bis hin zur Lichtquelle und Projektionsapparatur: Kein einziges Element dieser Kette von Artefakten, die zusammen den Film ausmachen und das Kino ermöglichen, wäre ohne die in Forschungslabors erzielten chemischen, physikalischen, elektrotechnischen, mechanischen, optischen, wahrnehmungspsychologischen sowie physiologischen Erkenntnisse realisierbar gewesen ${ }^{4}$.

Zweitens war der Film in die Produktion dieses wissenschaftlichen Wissens involviert. Schon vor dem Ersten Weltkrieg avancierten filmische Aufzeichnungen in verschiedenen akademischen Fachdisziplinen zum integralen Teil der Forschungspraxis: in der Psychiatrie, in den medizinischen Diagnosetechniken ganz allgemein, in der Chirurgie, der Psychologie, der Physiologie ebenso wie in der Arbeitswissenschaft und der Kriminalanthropologie. Die Beziehung zwischen Medien und Wissenschaft lässt sich über Jahrhunderte und Jahrtausende zurückverfolgen - doch die Fotografie und, ein halbes Jahrhundert später, der Film intensivierten und modifizierten diese Abhängigkeit. Als die Bilder ab Mitte der 1890er Jahre laufen lernten, dienten Bildmedien nicht mehr nur der optischen Dokumentation und visuellen Archivierung von physikalischen und chemischen Prozessen sowie Lebensvorgängen ${ }^{5}$, sondern fungierten als integraler Teil der Experimental-

$2 \mathrm{Zu}$ erwähnen sind hier auch Thesen einer grundsätzlichen Entgegensetzung von Kunst und Wissenschaft, so dass erstere als die Verneinung der letzteren erscheint. So schreibt Heide

Schlüpmann: «Die neue〈Kunst〉 des Films entsteht sogar aus dieser Verbindung [mit der Wissenschaft, J. T.]; daher wird leicht übersehen, wie ihm die Negation wissenschaftlicher Weltsicht zugleich innewohnt» (Schlüpmann 2001, 95).

3 Zur Verwissenschaftlichung der Industrieproduktion seit den 1880er Jahren und zur damals einsetzenden «second economic revolution» vgl. North 1981.

4 Crary 1996; Kern 1983. Vgl. auch: Kemner/Eisert 2000.

5 Krause/Pethes 2007. 
anordnung und der exakten Beweisführung. Visuelle Repräsentationen und sequentielle Aufnahmen vermochten auf diese Weise ihre genuin epistemische, eine erkenntnisfördernde und -erweiternde Rolle auszuspielen ${ }^{6}$.

Drittens war der Film seit seinen Anfängen ein wirksames Medium für die Distribution, Diffusion und für die Popularisierung wissenschaftlicher Erkenntnisse. Er verlieh den Resultaten schwer nachvollziehbarer Experimente eine faszinierende Sichtbarkeit und eine attraktive Anschaulichkeit. Der Film transponierte Daten aus dem Labor und Ergebnisse der Forschung in wissenschaftliche Geschichten, an denen sich die interessierte Öffentlichkeit staunend delektieren konnte. Der Film stellte die Fortsetzung der mit Broschüren und Zeitschriften um sich greifenden «Popularisierungsseuche» ${ }^{7}$ mit neuen technischen Mitteln dar. Umgekehrt hatten diese populären Darstellungen mit ihren Evidenzeffekten Rückwirkungen auf die Forschungsarbeit, indem sie die Hypothesenbildung anregten und Ideen für neue experimentelle Beweisführungen lieferten. Auf diese Zusammenhänge hat schon Mitte der 1930er Jahre der Wissenschaftsforscher und Mikrobiologe Ludwik Fleck hingewiesen. In seiner Studie über die «Entstehung und Entwicklung einer wissenschaftlichen Tatsache» sprach er generalisierend von den «allgemeinen Rückwirkungen populärer Wissenschaft» auf die Forschung und stellte fest: «Gewissheit, Einfachheit, Anschaulichkeit entstehen erst im populären Wissen; den Glauben an sie als Ideal des Wissens holt sich der Fachmann von dort. Darin liegt die allgemeine erkenntnistheoretische Bedeutung populärer Wissenschaft.» ${ }^{8}$ Zwischen wissenschaftlicher Forschungsdynamik und populärwissenschaftlichen Resonanzbedingungen gibt es - dies die These Flecks - einen Einfluss in beide Richtungen. Und den Bildmedien kommt - so lässt sich diese These weiterentwickeln - in dieser Wechselwirkung eine wichtige erkenntnistheoretische Relaisfunktion zu.

Diese dreifache Wechselwirkung von Film und Wissenschaft lässt sich seit den 1890er Jahren beobachten. Der Erste Weltkrieg markierte einen Einschnitt und zugleich eine Beschleunigung. Im fin de siècle hatten viele befürchtet, dass die wissenschaftlichen Produktivkräfte in präzedenzlose Destruktionspotentiale umschlagen könnten; im «Grossen Krieg» (wie die Zeitgenossen ihn nennen sollten) wurde diese Ahnung zur niederschmetternden Gewissheit. Gerade der Film bot in dieser Situation Raum für das effektvolle Exponieren kollektiver Ängste und öffentlicher Phobien. Die Synergien zwischen Wissenschaft und Film fanden eine Entsprechung in

6 Siehe z.B. Hoffmann 2006; diese epistemische Funktion schliesst nicht aus, dass im Film auch ein Medium für Hypnose und Suggestion gesehen wurde. Vgl. dazu Andriopoulos 2000.

7 Daum 2002, 2.

8 Fleck 1980, 152. 
der potenten Verbindung, die in den Kriegsjahren zwischen Krieg und Kino zustande kam. Das serielle Prinzip der Filmaufnahme wies eine technische Affinität mit dem Serienfeuer von Maschinengewehren auf; das Einfangen von Lichtquanten auf rasch aufeinanderfolgenden Zelluloidquadraten und das ratternde Ausstossen von Gewehrkugeln folgten derselben Logik der vollautomatisierten Repetition technischer Operationen in Sekundenbruchteilen. Paul Virilio, der dies in «Krieg und Kino» beschreibt, arbeitet zudem eine Homologie zwischen Flugzeug und Film heraus9. Die Flieger wurden als «fliegende Mirador» für Luftaufklärung und damit für eine neue Weise des Sehens eingesetzt; eine solche Revolutionierung des Blicks resultierte auch aus Filmvorführungen. Sowohl das Schlachtfeld wie das Kino stellen so Virilio - Wahrnehmungsfelder dar, auf denen Metamorphosen des Sehens stattfinden, welche den Blick der Menschen auf die Welt, in der sie lebten, nachhaltig veränderte ${ }^{10}$.

\section{Massenkultur und Wissenschaftspopularisierung im Film}

Der frühe (Kurz-)Film war ein «Cinema of Attractions» ${ }^{11}$ und als solches zunächst auf die Sensationen des Alltags oder herausragende Ereignisse gerichtet. Mit der film- und medienhistorischen Zäsur von 1905/1910, in der sich zunächst das stationäre Kino gegenüber der ambulanten Filmvorführung durchsetzte und dann der Spiel- oder Langfilm die Wirkungsästhetik des Kinos veränderte, brach eine neue Ära an. Schon vor dem Ersten Weltkrieg begann die grosse Zeit des «Kinos der Illusionen»; vorgeführt wurden soziale Dramen, in denen Erotik, Gewalt und Verbrechen für Spannung sorgten $^{12}$. Die Kriegsjahre lösten die angesprochenen Wahrnehmungsveränderungen aus, die in den 1920er Jahren breitenwirksam wurden. Nun stieg der Film zum privilegierten Medium für das Ausagieren affektiver Regungen und die visuelle Inszenierung von Massenphänomenen auf. Es machte sich eine neue Ökonomie der Aufmerksamkeit bemerkbar, in der Wunschund Schreckensvorstellungen spiegelbildlich aufeinander bezogen waren ${ }^{13}$. Eigentümlicherweise fand die Wissenschaft gerade in diesem emotional aufgeladenen Umfeld gute Resonanzbedingungen vor. Es wurden nicht nur zunehmend wissenschaftliche Erkenntnisse popularisiert, sondern die Vorstellungen der Wissenschaft und das Image des Wissenschaftlers wurden

9 Virilio 1986.

10 Virilio 1986, 13ff., 30ff., 72; Krause/Pethes 2007.

11 Gunning 1990.

12 Müller 2001, 62-65; Morat 2005.

13 Vgl. Franck 1998. 
im neuen Medium verändert. Die Fiktionalisierung der experimentellen Laborforschung alimentierte ambivalente technische Allmachtsphantasien, die auf Fortschritt und Zerstörung, auf Rettung und Untergang gleichermassen bezogen waren und in denen sich Identifikation und Abwehr merkwürdig mischen konnten. Diese affektive Aufladung der Wissenschaft förderte die Personalisierung von Emotionen in der Figur des Forschers; zugleich weitete sie den Horizont des Möglichen aus und bündelte normative Energien, die auf das Faktische zurückwirkten ${ }^{14}$.

Dieser Vorgang wurde - mit Blick auf die Fiktionsfabriken von Hollywood - seit den 1920er Jahren als «Amerikanisierung» diskutiert und perhorresziert. Er lief mit der Entfaltung eines massenkulturellen Mediensystems einher, in welchem die filmische Repräsentation wissenschaftlichen Wissens einen gesteigerten Stellenwert erhielt. Die Versuche, das öffentliche Interesse an der Wissenschaft zu erhöhen, und der Gebrauch von Forschungsergebnissen zu Unterhaltungszwecken wurden in ein Positivsummenspiel integriert. Das kommerzielle Kino nutzte die Wissenschaft für neue Plotstrukturen und Spezialeffekte und verwandelte sie in einen Generator für emotionale Spannung und Geistesblitze. In der Nachkriegszeit wurde die Verbindung von szientifischer Überraschung und cineastischer Unterhaltung enger und entwickelte sich mit dem seit den 1960er Jahren kometenhaft aufsteigenden Fernsehen in neue Dimensionen hinein. Es entstand eine Wissenschaft-Medien-Koppelung, die nun zu einem markanten Merkmal der politischen Öffentlichkeit wurde. Gerade das «bewegte Bild» akzentuierte das für diesen Verbund konstitutive Konkurrenzverhältnis von medialer Prominenz und wissenschaftlicher Reputation ${ }^{15}$. In diesem massenmedialen Unterhaltungssystem, das immer stärker den Regeln des Quotenwettbewerbs und der Kommerzialisierung unterliegt, kann sich die Medialisierung von Wissenschaft «weder in der Behandlung von wissenschaftlichen Themen, noch im Auftritt von Wissenschaftlern» erschöpfen ${ }^{16}$. In Anlehnung an Peter Weingart spricht Dirk Verdicchio von einem Kontinuum zwischen wissenschaftlicher Kommunikation und Populärkultur, welches es «unmöglich macht, die Grenze zwischen Wissenschaft und Popularisierung zu bestimmen ${ }^{17}$.

Für die Phase nach 1900 und vor allem für die Zwischenkriegszeit lassen sich verschiedene Momente dieses nach 1945 dominant werdenden Zusammenhangs bereits in Ansätzen erkennen. Ganz allgemein legitimierte sich

14 Vgl. Elsaesser 2002.

15 Weingart 2001, 239; vgl. auch Weingart 2005 und Schirrmacher 2008.

16 Verdicchio 2008.

17 Weingart 2001, 249f.; Verdiccio 2008, 55. 
die bürgerliche Klassengesellschaft auch über eine Bildungshierarchie, die einen abgestuften Zugang zu wissenschaftlichem Wissen voraussetzte und wiederum verstärkte. Auf die Strategie des Bildungsbürgertums, die eigene Position durch den intellektuellen Anschluss an die «Fortschritte» der Wissenschaft aufzuwerten, reagierte die Arbeiterbewegung mit Anstrengungen für den «Kulturaufstieg der arbeitenden Massen»; dabei wurde der grundlegend wissenschaftliche Charakter des Sozialismus betont, verbunden mit einem Plädoyer für eine anspruchsvolle Popularisierung eines wissenschaftsbasierten Weltbildes mit erzieherischer Wirkung. Biologisches Wissen erhielt einen hohen Stellenwert; mit der Tendenz zur Naturalisierung sozialer Phänomene korrespondierte der Aufstieg der Eugenik als einer weithin und auch in der politischen Linken akzeptierten Sozialtechnologie. Die Vorstellung, mit Hilfe der Wissenschaft könnten die gesellschaftlichen Konflikte, welche die kapitalistischen Industriegesellschaften des 19. Jahrhunderts prägten, befriedet werden, stiess weithin, auch klassenübergreifend, auf positive Resonanz ${ }^{18}$. Das frühe Kino nahm diese Tendenzen auf, wobei die Beziehungen zwischen Film und Wissenschaft disparat blieben ${ }^{19}$. Popularisierungsanstrengungen stützten sich vom «Masseneffekt» her noch immer primär auf illustrierte Broschüren, Zeitschriften und Bücher. Der Film versprach zwar die allgemeine Teilhabe an den neuesten naturwissenschaftlichen Erkenntnissen, doch diese Erwartung wurde wie schon bei den Printmedien durch soziale Distinktionsbedürfnisse durchkreuzt. Dennoch zeigten sich neue Entwicklungen. So nahm das Genre des Dokumentarfilms im Spannungsfeld von politischer Propaganda, sensationellem Reiseabenteuer, interest-Film und künstlerischer Ästhetisierung Kontur $a^{20}{ }^{20}$ Grosse Beachtung fand zum Beispiel die Serie Secrets of Nature, die 1922 unter der Regie von H. Bruce Woolfe startete und bis 1933 fast 150 Filme umfasste. Diese Serie überspielte naturwissenschaftliche Ansprüche mit der Verführung durch das Dokumentarische. Es ging um das Faszinosum des Verborgenen in den Mikro- und Makrowelten der Natur, das durch filmische Aufnahmetechniken für ein breites Publikum zugänglich gemacht werden konnte. Die anthropomorphe Darstellung von biologischen und physikalischen Vorgängen und von Lebewesen steigerte das Identifikationspotential und den Unterhaltungswert der Filme. Diese kommunizierten die Botschaft, dass die Natur ein weites Reich für Überraschungen und Entdeckungen sei - und damit waren sie auch geeignet, die Akzeptanz der experimentellen Forschung zu erhöhen.

18 Vgl. Tanner 2007.

19 Ross 2006.

20 Von Keitz et al. 2001. 
Eine anders gelagerte Ambivalenz und Durchlässigkeit zeigt sich etwa in den vielen Aufklärungsfilmen, die vereinzelt schon seit der Jahrhundertwende den Kampf gegen die Geschlechtskrankheiten zum Thema machten und die in den 1920er Jahren boomartig aufkamen. Gegen Ende der roaring twenties rekurrierten allerdings viele Filme dieses Genres auf das volksgesundheitliche Anliegen vor allem deshalb, weil dies die einzige tolerierte Möglichkeit war, tabuisierte erotische und sexuelle Szenen auf die Leinwand zu bringen. Aufgrund dieses Verdachts gerieten aufklärerische Popularisierungsstreifen ins Visier öffentlicher Sittenwächter und erfreuten sich umgekehrt steigender Beliebtheit ${ }^{21}$. So stiess auch der 1931 von der Schweizerischen Gesellschaft zur Bekämpfung der Geschlechtskrankheiten in Auftrag gegebene und unter der Regie von Walter Ruttmann produzierte Kinofilm «Feind im Blut», der durch eine avantgardistische visuelle Umsetzung Furore machte, auf enorme Vorbehalte, nicht nur in konservativen Kreisen ${ }^{22}$. Verallgemeinernd kann festgestellt werden, dass der Kinofilm in der Zwischenkriegszeit mit neuen Vergnügungsformen Erfolge feierte. Es war das rasch aufstrebende Unterhaltungskino, welches mit seinem kommerziellen Kalkül des raschen «Einspielens» der Auslagen für die Filmproduktion die Formen, Chancen und Grenzen wissenschaftlicher Popularisierung dominierte.

\section{Verrückte Wissenschaftler und wissenschaftliche Experten}

Diese Akzentverlagerung in den volkshygienisch-medizinischen Popularisierungsprojekten in Richtung eines verbotenen Publikumsgeschmacks war bloss eine Facette des unaufhaltsamen Aufstiegs von Historienfilmen und Kinovorstellungen mit Familiendramen, dystopischen Sensationsgeschichten und Horrorshows. Die mediale Selektionslogik, die auf die «breiten Massen» ausgerichtet war, und die ästhetisch-technischen Eigenschaften des filmischen Mediums, das magische Szenen und wunderbare Ereignisse scheinbar mühelos auf die Leinwand bringen konnte, ergänzten sich gegenseitig in einer Eskalation des Spektakulären. Mit diesem medialen Wandel, der durch den Aufstieg des Films als eines Mediums für erschwingliche und spannungsgeladene Massenunterhaltung ausgelöst wurde, war eine Metamorphose der Figur des Wissenschaftlers verbunden. War im 19. Jahrhundert auch in der populären Version des Forschers die Idee einer heldenhaften Auf- 
opferung für eine grosse wissenschaftliche Idee ( $«$ Jede grosse Entdeckung erfordert ein Menschenleben») massgeblich, so rastete im Film der ersten Jahrzehnte des 20. Jahrhunderts dieses szientifische Objektivitätsideal aus. Der «verrückte Wissenschaftler» stieg zur cineastischen Ikone auf; sein Attraktionswert begann Sexualbeziehungen und Erotikszenen zu überflügeln. Auch wenn die Topoi vom mad scientist und man-made monster dann in den Jahrzehnten nach dem Zweiten Weltkrieg ihre Virulenz und Symptomatik wiederum verloren, waren sie in den beiden Jahrzehnten nach dem Ersten Weltkrieg zentral23.

In der Studie «Mad, Bad and Dangerous?» untersucht Christopher Frayling die spannungsreiche Beziehung zwischen der Figur des Naturforschers und dem Medium des Films in der aufstrebenden Kulturindustrie, die Wissenschaft nunmehr als populäres Faszinosum vermarktet ${ }^{24}$. Wie vor ihm schon Andrew Tudor in «Monsters and Mad Scientists» und David Skal in «Screams of Reason» ${ }^{25}$ zeigt auch Frayling, wie häufig und wie sehr Filmplots den modernen Forscher als mad scientist in Szene setzen, dessen höchste Ambition die Schaffung eines menschlichen Monsters ist. Das Experimentieren im Labor wurde dabei als veritable «Rocky Horror Picture Show» aufgezogen, in der Entertainment und Schrecken eine merkwürdige Mischung eingingen ${ }^{26}$. Der Wissenschaftler mutiert vom intellektuellen hero, der sich zum Wohle der Menschheit abmüht, zum irren Genietypen, der die Entfaltung destruktiver Potenzen gleichsam als wissenschaftliche Selbstverwirklichung praktiziert.

Es ist davon auszugehen, dass das neue verführerische Angebot auf eine durchaus selbstbewusste Nachfrage stiess. Filmzuschauer und Kinobesucherinnen waren (und sind) keine passiv-rezeptiven Wesen, die Phantasmen und Phantasmagorien aus Wissenschaft und Technik auf sich einwirken und sich verängstigen lassen. Sie nutzen vielmehr Angebote nach ihren eigenen, eigensinnigen Vorstellungen. Es ist auch für die Zwischenkriegszeit sinnvoll, ein «double viewing ${ }^{27} \mathrm{zu}$ unterstellen, das heisst, eine Rezipientenhaltung, die gleichzeitig zwei gegenläufige Bedürfnisse befriedigt: Da ist zum einen der Hang zum Fiktionalen. Dieser legt es nahe, sich emotional voll in eine Filmstory zu investieren, das Identifikationsangebot und das Gefühlsrepertoire auszuschöpfen, sich im dunklen Kinosaal mitnehmen zu lassen und

23 Für diese Diagnose vgl. Verdicchio 2008; für die Kritik am mad scientist vgl. Weingart 2005, $189 \mathrm{ff}$.

24 Frayling 2002.

25 Tudor 1989; Skal 1998.

26 Weingart 2003; vgl. auch Weingart 2005, $189 \mathrm{ff}$.

27 Verwoert 2003. 
etwas Ausserordentliches zu erleben. Zum anderen gibt es den Willen zur Realität, der einen dann doch nicht vergessen lässt, dass es sich «nur um einen Film» handelt, um eine durch Bildsequenzen erzeugte Illusion also. Die Überraschungseffekte und die vielfältigen, von Angst, Brutalität, Freude, aber auch von Humor, Ironie durchsetzten Stimmungslagen stellen gleichsam den emotionalen Gegenwert zum Eintrittspreis dar. Wobei das Vergnügen, das ein solches cineastisches Erlebnis vermittelt, sich dann durch das Mitredenkönnen über bekannte, berühmte Filme in die soziale Kommunikation hinein verlängern kann.

Eine solche Haltung lässt sich in fast allen dystopischen Filmen, die mit wissenschaftlichen Projektionen arbeiten, konstatieren. Philipp Sarasin hat in einem Beitrag zur Wirkungslogik der Populärwissenschaft darauf hingewiesen, dass die perverse Macht des mad scientist dem Publikum gleichzeitig eine Identifikationsmöglichkeit anbietet und es ihm dadurch erlaubt, «sich schauernd mit dem obszönen Geniessen desjenigen [zu] identifizieren, der das Geheimnis kennt» ${ }^{28}$.

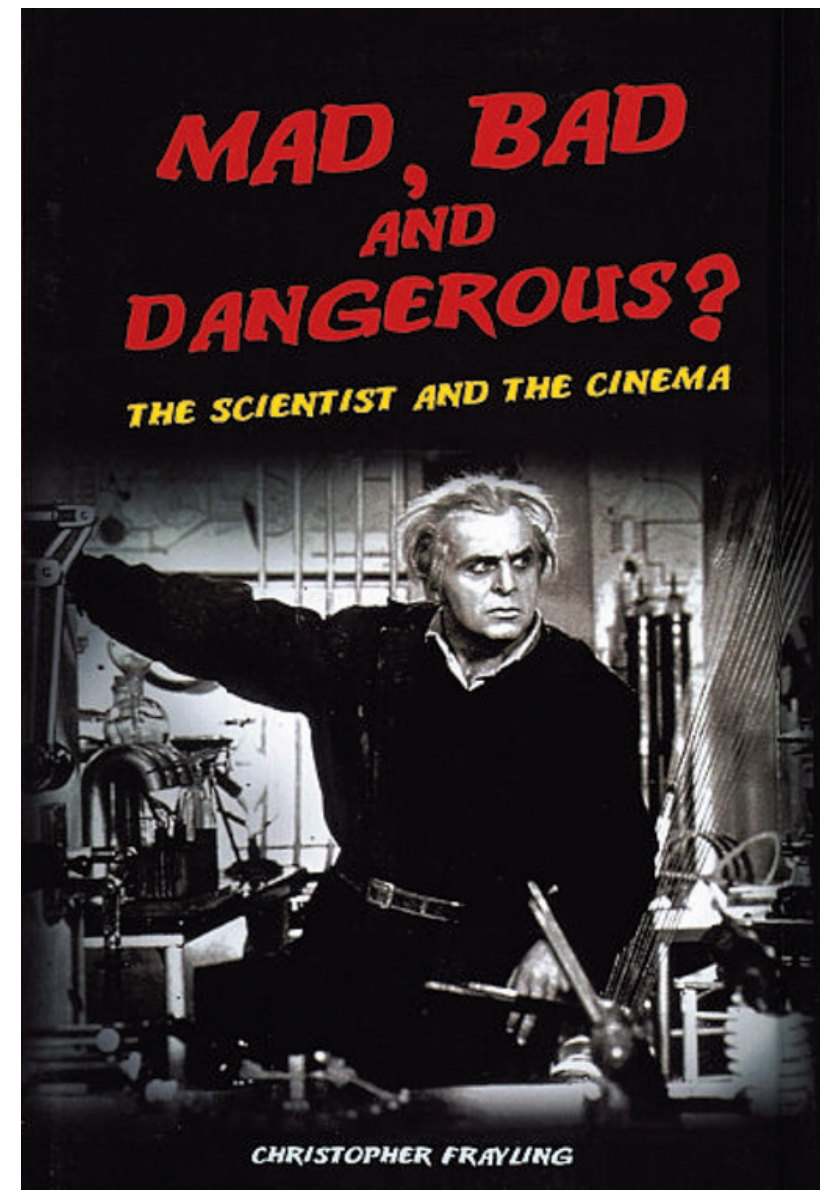

Abb.1. Der verrückte Erfinder Rotwang in Fritz Langs «Metropolis» (1927).

28 Sarasin 2003, 254. 
Genau darum geht es beim genialen Erfinder Rotwang (gespielt von Rudolf Klein-Rogge), der auf dem Frontumschlag von Fraylings Buch abgebildet ist. Dieser «verrückte Wissenschaftler» spielt in Fritz Langs 1927 uraufgeführtem Film «Metropolis» eine der Hauptrollen ${ }^{29}$. Der irre, aber keineswegs wirre Forscher schafft im wissenschaftlich-technischen Akt ein weibliches Maschinenwesen, programmiert es aber aus verletzten Liebesgefühlen um. Emotionen verrücken die Szene: In Rotwangs Augen blitzt bereits die beabsichtigte Fehlfunktion auf - und tatsächlich wird sich das neugeschaffene Techno-Wesen, das den Arbeitssklaven im Untergrund die Unabänderlichkeit und Ausweglosigkeit ihres Schicksals einhämmern soll, gegen die Absichten des Auftraggebers richten und die Proleten zur offenen Revolte gegen die metropolitane Bourgeoisie aufstacheln. Langs Parabel der eruptiven Spannungsmomente in einer kapitalistischen Klassengesellschaft hat ihren Ausgangspunkt in einem pervertierten Laborexperiment. Rotwang steht im Moment seiner Kreation eine jouissance ins Gesicht geschrieben, die dem Zuschauer das Gefühl geben kann, am Geheimnis der Macht dieses Wissens teilzuhaben, so dass der mad scientist vom unheimlichen «Anderen» in etwas seltsam Eigenes transformiert wird und auf diese Weise durch den Kinobesucher affektiv anverwandelt werden $\mathrm{kann}^{30}$. Die Kaskaden von Katastrophen, die im Film durch angewandte Forschung ausgelöst werden, vermitteln keine Botschaft nach dem Modus der Aufklärung, die vor möglichen Folgen einer zunehmend verwissenschaftlichten Gesellschaft warnt, sondern ermöglichen es dem Zuschauer, sich an der Erwartung geballter Schrecklichkeiten zu erfreuen.

So fungiert das Kino der Zwischenkriegszeit als imaginärer Verstärker, als Gefühlsgenerator, der nicht nur die Wunschenergien des Publikums zum Fliessen oder, besser: zum Überfliessen bringt, sondern auch die Bedrohungsphantasien der Zuschauer/-innen bewegt ins Bild setzt und diese dadurch zu dem fusioniert, was man damals die «cineastische Masse» genannt hat $^{31}$. Die Imaginationen der Zuschauermassen weisen allerdings selber einen Zeitindex auf. Machen sich heute eingebildete Menschheitserretter oder apokalyptische Monster im Labor an Genen und Klonen zu schaffen, so musste Anfang des 19. Jahrhunderts Dr. Victor Frankenstein noch selber Hand an Tiere und Menschen legen, um zu den Ingredienzien und Versatzstücken seines Monsters zu kommen. Frankenstein hat dann rasch ein -

29 Vgl. Elsaesser 2000. Im Sommer 2008 tauchte in einem Filmmuseum in Argentinien die Berliner Premierenfassung auf, die um 30 Minuten länger ist als die gekürzte Exportversion des Films. Vgl. Kasten 2008.

30 Diese Interpretation folgt dem Vorschlag von Philipp Sarasin 2003.

$31 \mathrm{Vgl}$. Gamper/Schnyder 2007. 
noch immer brauchbares - Vorbild abgegeben, das der Nemesis der life sciences ein Gesicht verlieh. Der «moderne Prometheus», wie ihn Mary Shelley 1818 in die literarische Welt entlassen hat, ist ein einprägsames Beispiel im doppelten Sinne: zum einen übertrug sich der Name des unglücklichen Schöpfers der monströsen Kreatur auf diese letztere, so dass die Erinnerung an ihn fortan im Anrufen der Kreatur weiterlebt. Zum anderen wurde ein in der Romanvorlage durchaus ansehnliches, mit menschlichen Zügen versehenes und damit empathiefähiges Wesen durch seine filmische Inszenierung zum technoiden Schocker ${ }^{32}$. Als Frankenstein im Jahre 1910 in den New Yorker Edison Studios unter der Regie von J. Searle Dawley zum ersten Mal verfilmt wurde, war er noch in der Lage, über sein eigenes Erscheinungsbild zu erschrecken und an seinen Gefühlen zu verzweifeln; die gleichnamigen Filme der Zwischenkriegszeit machten dann aus dem Monster die Hauptfigur von Horrorfilmen, die darauf angelegt waren, den zahlenden Zuschauer dem restlosen Wahnsinn auszusetzen, auf dass er sich möglichst gut unterhalte.

Neben und in vielem auch gegenläufig zu diesem Trend zum filmtechnisch avancierten Entertainment liess sich in der Zwischenkriegszeit eine andere Entwicklung konstatieren: der Aufstieg des visuell komplex argumentierenden wissenschaftlichen Dokumentar- und Lehrfilms. Dies geschah unter veränderten innerwissenschaftlichen und gesellschaftlichen Vorzeichen. Das Zeitalter der kapitalistischen Industrialisierung hatte den Triumph der Technik gefeiert und stand im Banne eines ungebrochenen Fortschrittsglaubens. Auch noch im fin de siècle inszenierten Zeitschriften und repräsentative Bücher die grossen technisch-industriellen Errungenschaften - Kanal- und Tunnelbauten, Eisenbahnlinien, Elektroinstallationen und Verkehrsmittel wie Ozeandampfer und Dampflokomotiven - in visuellen Darstellungen. In den Anfängen des Films wurden solche Phänomene auch auf Zelluloid gebannt. Im beginnenden 20. Jahrhundert hatte sich allerdings der $\mathrm{Zu}$ kunftshorizont durch Dekadenzängste und Degenerationsbefürchtungen verdüstert. Diese Einbusse an Gewissheit hatte ein Pendant in der Wissenschaft: Hier wich der robuste Kausaldeterminismus einem Wahrscheinlichkeitsdenken und einer Relativierung bisher fester Bezugsgrössen. Dies hatte Konsequenzen für die Popularisierung der Wissenschaft. Die Einstein'sche Relativitätstheorie und eine vererbungstheoretisch informierte Darwin'sche Evolutionstheorie, wie sie aus der «grossen Synthese» der 1930er und 1940er Jahre hervorging, liessen sich weniger gut trivialisieren als linear wirkende Naturgesetze und sozialdarwinistische Gesellschaftsmodelle. Es gehört zu

32 Vgl. Turney 1998. 
den Spezifika der Phase nach dem Ersten Weltkrieg, dass die Komplexitätssteigerung innerhalb der Wissenschaft die Ansprüche an die Wissensvermittlung erhöhte. Es entstand ein neuer Typus von Film, der sich deutlich gegenüber der gleichzeitig aufkommenden science fiction sowie den madscientist-Filmen abgrenzte und der sich auch vom sozialmoralisch dramatisierenden «Aufklärungsfilm» unterschied. Spielen letztere mit der Faszination vor dem Schrecken oder den Verheissungen der modernen Gesellschaft und ihrer Wissenschaft, so appellierte der anspruchsvolle Popularisierungsfilm an die Neugierde interessierter Laien, denen der Eindruck vermittelt wurde, dass sie dank des visuellen Nachvollzugs abstrakter Theorien an den neuesten, vielfach kontraintuitiven Erkenntnissen der Wissenschaft teilhaben können. Dabei setzten die Produzenten auf den Unterhaltungswert von Aha-Erlebnissen und alltagsweltlichen Paradoxa; sie stellten zugleich die immaterielle Gratifikation in Aussicht, welche mit der Teilhabe am Wissen einer technischen Gesellschaft verbunden war. Anders als im kommerziell einschlägigen Kinofilm, in dem Wissenschaftler, wenn sie in einer Hauptrolle wirkten, als Garanten für action und bösartige Überraschungen fungierten, legte der populäre Wissenschaftsfilm Wert auf Fachexpertise, sei es durch die direkte Involvierung von renommierten Mitgliedern der scientific community in der Darstellung der Sachverhalte, sei es durch die Einrichtung des filmbegleitenden Vortrags, der durch einen ausgewiesenen Spezialisten gehalten wurde. Dieses wissenschaftsdokumentarische Filmgenre wird im Folgenden anhand von zwei Beispielen - der Verfilmung der Einstein'schen Relativitätstheorie in den frühen 1920er Jahren und der filmischen Popularisierung des Darwinismus im Jahre 1937 - dargestellt.

\section{«Die Grundlagen der Relativitätstheorie»: Grenzen der filmischen Darstellung}

Nach dem Ersten Weltkrieg wurden von unterschiedlicher Seite die Versuche intensiviert, die anspruchsvolle und dem Alltagsverstand wenig zugängliche, durch die elegante und schlichte Formel $\mathrm{e}=\mathrm{mc}^{2}$ gleichzeitig weithin faszinierende Relativitätstheorie einem breiteren, gebildeten Publikum zu vermitteln ${ }^{33}$. In den Jahren nach 1920 erschien eine Einführung nach der anderen; diese Broschüren zielten darauf ab, Interessierten einen Einblick in den state of the art in der Physik zu geben. Gegen diese Popularisierungsbestrebungen schrieben Gegner der Relativitätstheorie an. Prominent war

33 Eisenstaedt 2006; Shepherd-Barr 2006. 
vor allem Ernst Gehrcke, Mitarbeiter und ab 1926 Abteilungsleiter an der Physikalisch-Technischen Reichsanstalt in Berlin, der eine umfangreiche Pressedokumentation führte ${ }^{34}$. Von dieser wissenschaftlich intendierten Kritik lassen sich antisemitische Ressentiments nur schwer unterscheiden. 1922 sah sich der damals in Berlin lebende Albert Einstein nach der Ermordung Walther Rathenaus mit Morddrohungen konfrontiert, die durch rabiate Judenfeindschaft motiviert waren ${ }^{35}$. Als bekannt wurde, dass Einstein im selben Jahr auf der Hundertjahrfeier der Gesellschaft Deutscher Naturforscher und Ärzte in Leipzig den Festvortrag halten sollte, lancierten seine akademischen Gegner eine geharnischte Protesterklärung, in der sie die Relativitätstheorie als «unbewiesene Hypothese» und «unhaltbare Fiktion» denunzierten. Der Text fährt fort: «Die Unterzeichneten betrachten es als unvereinbar mit dem Ernst und der Würde deutscher Wissenschaft, wenn eine im höchsten Masse anfechtbare Theorie voreilig und marktschreierisch in die Laienwelt getragen wird [...].» ${ }^{36}$ Die Kampagne wirkte; Einstein sagte seinen Auftritt ab. Die Propaganda gegen seine wissenschaftlichen Leistungen hielt indessen an. Im November 1923 schrieb der Arzt und Schriftsteller Alfred Döblin, der mit seinem «Berlin Alexanderplatz» weltberühmt werden sollte und später in den USA vom Judentum zum Katholizismus konvertierte, im «Berliner Tagblatt» über die «scheussliche Relativitätstheorie» Folgendes:

Wir werden durch die Scheinweisheiten, den Papierfortschritt der Mathematiker von den wichtigsten Quellen des Lebens abgedrängt. Die Relativitätstheorie etwa wird von Millionen Gebildeter teils nicht begriffen, teils wissen sie nicht, was sie damit anfangen sollen. Wer aber ist es, der sie dazu drängt, die Lehre so überhaus ernst zu nehmen? Die Hierarchie der Wissenschaftler, der Geheimbund, die Verschwörung und Freimaurerei der Mathematiker.

Döblin fügt dann an:

Ach Gott, liebe Kinder, lass die Damen und Herren ihre Verschwörung machen. [...] Es gibt andere bessere, tiefere, reichere Wege sich der Natur zu nähern. Wir wollen uns unsere einfachen Gedanken und unseren graden Gang von niemandem nehmen lassen. Die Natur ist wirklich unsere Mutter: wie sollte nicht jedes, jedes Kind seine Mutter erkennen. ${ }^{37}$

Angesichts dieser Widerstände und Schwierigkeiten versuchten Anhänger der Relativitätstheorie schon 1919, als eine von der Allgemeinen Relativitätstheorie prognostizierte Lichtablenkung bestätigt wurde, einen Lehrfilm

34 Milena Wazeck erwähnt 2000 Zeitungsartikel und spricht von weiteren 3000 während des Zweiten Weltkrieges verschollenen Beiträgen. Vgl. Wazeck 2005a, 49; vgl. auch Wazeck 2005b. Die Gehrcke-Dokumente sind auf der Website des Max-Planck-Instituts (MPI) für Wissenschaftsgeschichte einsehbar. Vgl. http://echo.mpiwg-berlin.mpg.de/content/modernphysics/gehrcke/ newspaper_articles/gehrcke_folders (15.04.2008).

35 Vgl. Wazeck 2005c, 222ff.

36 Renn 2005, 363.

37 Renn 2005, 365. 
zu produzieren. Dass Albert Einstein sich nicht für den Film engagieren wollte, hängt primär mit den permanenten deutschnationalen und antisemitischen Angriffen zusammen, denen er sich ausgesetzt sah. Er wollte aber auch generell nicht mit einem Film in Verbindung gebracht werden. So entwickelte Hanns-Walter Kornblum, Abteilungsleiter des Wissenschaftsfilms der Deutschen Lichtbild-Gesellschaft (Deulig), ein Filmprojekt in Eigenregie und realisierte dies in der neugegründeten Colonna $\mathrm{GmbH}$, einer Gesellschaft für die Herstellung von Lehrfilmen. Nach drei Jahren Produktionszeit, in denen die Zeichner fast 80000 Einzelbilder anfertigten, konnte der ästhetisch und formal avantgardistische Film 1922 fertiggestellt werden ${ }^{38}$. Das Resultat wurde von vielen Experten und Laien als eine Sensation empfunden. Heute gilt die Originalversion als verschollen; erhalten ist einzig eine englische Kurzfassung aus dem Jahre $1923^{39}$. Diese zeigt plastische Beispiele, die der Stummfilm bieten konnte, so z.B. einen Lastkahn in einem Kanal, der von einem rückwärts darauf gehenden Seemann, der einen Stab auf Grund gesetzt hat, vorwärts geschoben wird. Vom Boot aus gesehen, bewegt sich der Mann, vom Ufer aus betrachtet das Boot. Die Zuschreibung von Bewegung erweist sich als Frage des Beobachtungsstandpunktes, alle Aussagen sind relativ. Später wird eine grosse Kanone vorgeführt, die ein Objekt ins Weltall feuert, welches wiederum unterschiedliche Perspektiven demonstriert. Es gibt auch Leuchttürme, die Strahlen auf ein Luftschiff richten. Und ein rasch drehendes Rad, auf dem diametral versetzt zwei in dieselbe Richtung zielende Pistolen montiert sind, die gleichzeitig auf eine Leinwand feuern. Während der Lichtblitz dort gleichzeitig zu sehen ist, treffen die beiden Kugeln zu unterschiedlichen Zeiten ein, weil das Umlauftempo des Rades zur Geschwindigkeit der einen Pistole addiert, während sie bei der anderen subtrahiert werden muss. Mit der komplexen theoretischen Überlegung verbindet sich eine leicht nachvollziehbare Versuchsanordnung, die dann auf die Erde übertragen werden kann. Weil sich diese ebenfalls wie ein Rad um die eigene Achse dreht, treffen die mit derselben Geschwindigkeit auf den Mond abgefeuerten Geschosse dort zu unterschiedlichen Zeiten ein, im Gegensatz zum Licht, das wiederum simultan zu sehen ist.

Der Film wurde mit beträchtlichem Aufwand lanciert und bestach durch seine technische Raffinesse. Die Filmkritik indes war gespalten. Die «Frankfurter Zeitung» schrieb am 3. April 1922 von einem «ungewöhnlich starken Besuch»; nach dem zweiten Teil habe jedoch eine «allgemeine Flucht» ein-

38 Wazeck 2005b, 25.

39 Die Filmkopie befindet sich auf der CD in der Publikation: Max Planck-Institut für Wissenschaftsgeschichte Berlin, Pocketguide: Albert Einstein. Chief Engineer of the Universe (Berlin 2005). 


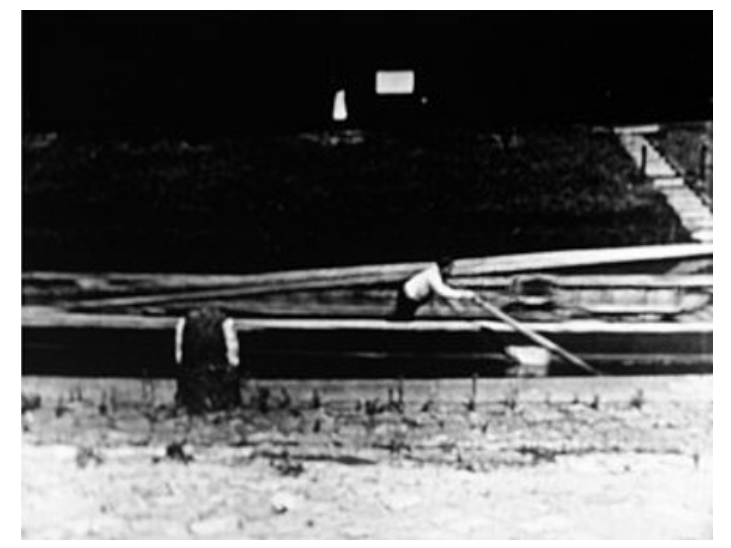

a

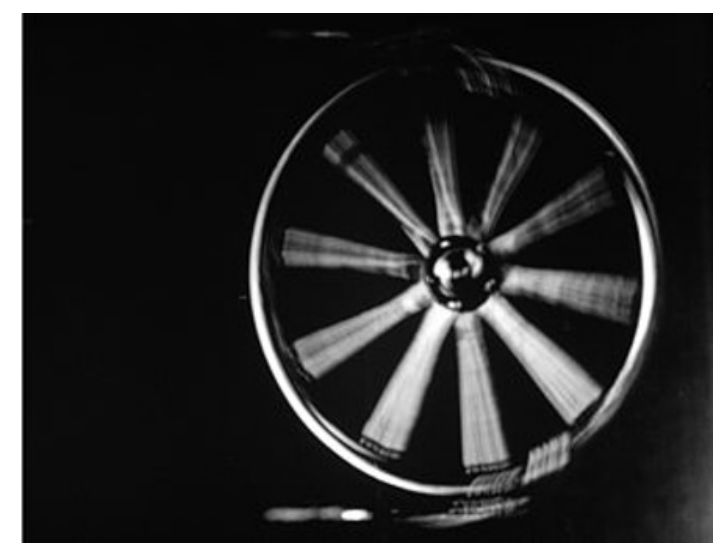

$\mathrm{c}$

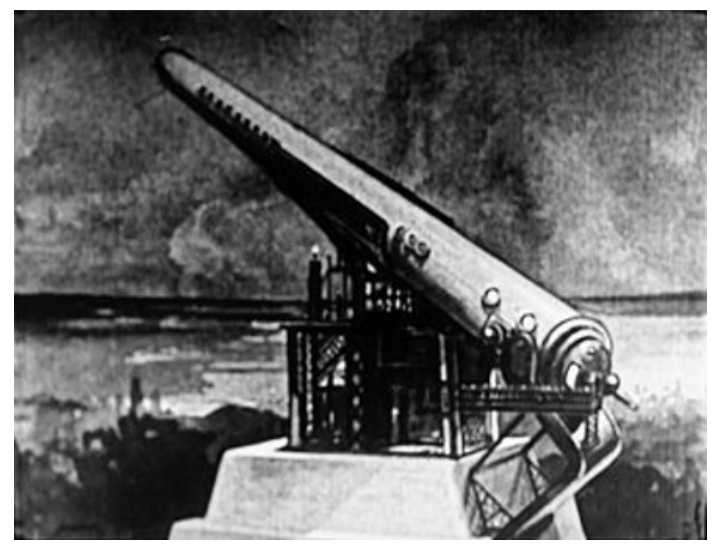

b

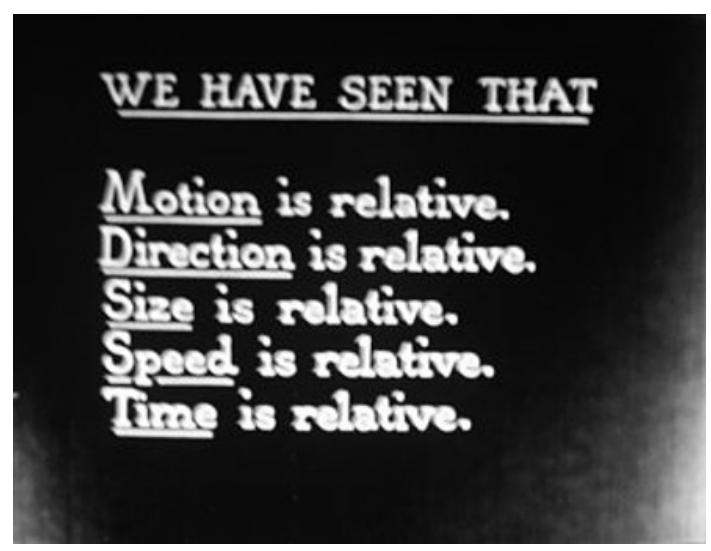

d

Abb. 2. Szenen aus dem Film «Die Grundlagen der Einsteinschen Relativitätstheorie» (1922). (a) Ein Kahn bewegt sich relativ zum Ufer. (b) Beginn einer Reise im All. (c) Schussrad: Das Licht der beiden Pistolenschüsse kommt, im Unterschied zu den Kugeln, gleichzeitig auf der Zielscheibe an. (d) Alles ist relativ - ausser die Lichtgeschwindigkeit.

gesetzt, was die Zeitung als «doppelt beschämend» qualifizierte, denn zum einen zeuge ein solches Verhalten generell von fehlender Neugierde und zum anderen seien vor allem «Pressevertreter» zur Visionierung eingeladen worden $^{40}$. Auch der den Film insgesamt lobende Rezensent kritisierte allerdings die «besonders im dritten Teil störende starke Schwerverständlichkeit des Vortragenden, die zwar durch seine Indisposition entschuldigt war, aber nicht verhindern konnte, dass lange Zeit über nur Geräusche, aber keine Worte von den Ohren der Zuhörenden aufgenommen wurden. Möglich, dass an dieser Störung auch allein die ungeschickte Aufstellung des Vorführungs-

40 Alle Zeitungsbeiträge sind nach der Gehrcke-Sammlung am MPI für Wissenschaftsgeschichte zitiert. Vgl. Internet-Adresse in Fussnote 34. 
apparates schuld war, dessen starkes Geräusch oft den Vortragenden übertönte.» Die Beantwortung der Frage, wie weit die Relativitätstheorie durch den Film angemessen dargestellt werde, war nicht einheitlich. Im «Filmkurier» vom 4. April 1922 wurde dazu bemerkt: «Jedenfalls gibt es kein anderes Mittel, irgend einem Menschen die Relativitätstheorie anschaulicher zu machen.» Tags darauf konstatierte die Zeitung «Germania» eher enttäuscht, dass da, «wo der 2000 Meter lange Film aufhörte», Einsteins Theorie «erst angefangen» hätte. Gezeigt worden seien nur «mehr oder minder bekannte Tatsachen und Vorkenntnisse», was jene enttäuscht habe, «die zu diesem Film wie zu jedem anderen kamen und die einen erläuternden Vortrag als ein Attentat gegen ihre eigene souveräne Vernunft oder doch als eine Störung des angenehmen musikalischen Film-Dunkels empfinden ${ }^{41}$.

Unter formalen Gesichtspunkten sind die Homologien zwischen der Theoriekonstruktion und der filmischen Visualisierungslogik frappant. Aufgrund dieser strukturellen Vorstellungs- und Darstellungsübereinstimmung lässt sich die Problemstellung der Relativitätstheorie besonders eindrücklich im filmischen Medium entwickeln. Damit wird das theoretische Wissen allerdings transformiert; es konkretisiert sich gleichsam aus seiner mathematischen Form heraus und wird zum Exempel; dieses funktioniert, weil - wie dies etwa in der Filmtheorie des russischen Formalismus und in anderen theoretischen Ansätzen zwischen der Jahrhundertwende und den 1920er Jahren herausgearbeitet wurde - in der filmischen Repräsentation die räumlichzeitliche Kontinuität wegfällt; aufgrund der Relativierung der Raumkoordination und der Fragmentierung der Zeit kann die Montage Gestaltungsspielräume öffnen; auf elegante Weise lassen sich so Perspektivenwechsel und Grössenveränderungen realisieren. Anders gesagt: Temporal- und Raumkonstruktionen können nahezu beliebig durch Zeitlupe und Zeitraffer bzw. durch Dehnung und Komprimierung verändert werden; der Film stellt in diesem Sinne ein visuelles Probehandeln dar, in dem sich die Widerständigkeit der Materialität ausschalten und spielerisch manipulieren lässt. Damit eröffnen sich nicht nur publikumswirksame Einsichten, sondern die experimentellen Operationen vermitteln auch neue, wissenschaftlich relevante Erkenntnisse. Darin besteht die epistemologische Funktion des Films.

41 Weitere Zitate sind auch unter folgender Internet-Adresse zu finden: http://www. kinematographie.de/EINSTEIN.HTM+colonna+film+geschichte $\& \mathrm{hl}=\mathrm{de} \& \mathrm{gl}=\mathrm{ch} \& \mathrm{ct}=\mathrm{clnk}$ $\& \mathrm{~cd}=1(15.4 .2008)$. 


\section{«Images mathématiques ...» (1937): Kampf um den Darwinismus im Film}

Beim zweiten Exempel lassen sich weniger Spannungen zwischen Film und (politischer) Öffentlichkeit denn innerwissenschaftliche Kontroversen identifizieren. Es handelt sich um einen Lehrfilm zur Popularisierung des (Neo-)Darwinismus, der während der Volksfrontregierung in Frankreich entstanden war. Die Politisierung darwinistischen Gedankenguts war damals - sei es in Form eines von Herbert Spencer inspirierten «Sozialdarwinismus» oder als «proletarischer Biologismus» $»^{42}$ - weit verbreitet ${ }^{43}$. In dieser Phase gab es Anstrengungen, von einem ideologisierten «Kampf ums Dasein» im Namen Darwins wegzukommen und die Evolutionstheorie auf mathematischer Grundlage jenseits gesellschaftlich-kultureller Kurzschlüsse zu reformulieren. Für diese Intention stand das Programm der «Great Synthesis», das die Darwin'sche Theorie mit der Mendel'schen Vererbungslehre vereinigte.

1937 drehte der erfolgreiche französische Dokumentarfilmproduzent Jean Painlevé (1902-1989) in Zusammenarbeit mit Achille-Pierre Dufour den Film «Images mathématiques de la lutte pour la vie», der diesen neuesten Entwicklungsstand der Evolutionstheorie vorführen sollte ${ }^{44}$. Painlevé war 1930 an der Gründung der «Fédération internationale du cinéma scientifique» beteiligt gewesen und stand dem damals ebenfalls neueingerichteten «Institut français du cinéma scientifique» als Direktor vor ${ }^{45}$. Painlevé, der stark durch die ästhetische Avantgarde beeinflusst war und die Experimente des abstrakten Films rezipierte, drehte zwischen 1926 und den beginnenden 1980er Jahren mehr als 200 Filme und entwickelte dabei seit den 1930er Jahren eine besonders populäre Kombination von fantastischer Darstellungsform und wissenschaftlicher Dokumentation ${ }^{46}$. Der Titel des 1937er Films lehnte sich an eine Publikation des italienischen, in Rom lebendem Mathematikers Vito Volterra an, der konzeptionell und beratend

42 Mocek 2002, 33.

43 Während die einen Wissenschaftshistoriker, z.B. Reinhard Mocek, die «weltanschauliche Sprengkraft des Darwinismus» hervorheben (Mocek 2002, 166), relativieren andere Historiker wie Peter J. Bowler «die Originalität und Dominanz Darwins für die Biologiegeschichte» (zitiert nach Daum 2002, 21).

44 Den Hinweis auf diesen Film verdanke ich Ariane Tanner, die an einer Untersuchung zur Geschichte der Populationsdynamik und zur Mathematisierung der Biologie, insbesondere zur Genese der Lotka-Volterra-Formel, arbeitet. Vgl. http:/www.wiss.ethz.ch/pfw/ personen_tanner.html.

45 Boussinot 1995, Bd. 2, 1574ff.; Bawden 1977, 579.

46 Vgl. die arte-Filmdokumentation zu Jean Painlevé: http://www.arte.tv/de/film/FilmstarWissenschaft/Fotoalbum-Dreharbeit-von-J-Painleve/Die-phantastischen-Filmweltendes-Jean-Painleve/1297226.html (15.4.2008). 
mitwirkte $^{47}$. Das Szenario steuerte der aus Russland stammende Biologe Vladimir A. Kostitzin bei ${ }^{48}$. Auftraggeber war Painlevés Institut, das den Film 1937 zur Eröffnung des im «Grand Palais» untergebrachten «Palais de la Découverte» vorführte. Der Film wurde durch mathematische Grafiken animiert, mit denen theoretische Ideen demonstriert werden konnten; er führte die Zuschauer auch durch Laboratorien und in die Natur, wo die Populationsdynamik zwischen mehreren Arten beobachtet wurde. Untersucht wurde das anspruchsvolle Problem der Wahrscheinlichkeit, mit der die natürliche Selektion unter wechselnden Umweltbedingungen unterschiedliche Resultate hervorbringt ${ }^{49}$.

Während der Entstehung dieses Films kam es zu einer signifikanten Kontroverse zwischen Volterra und seinem Schwiegersohn, dem Zoologen und Meeresbiologen Umberto D'Ancona. Volterra gehörte Mitte der 1920er Jahre zusammen mit dem zeitgleich, jedoch unabhängig publizierenden amerikanischen Chemiker und Statistiker Alfred James Lotka zu den Begründern einer mathematischen Biologie; die Lotka-Volterra-Formeln gehören heute zum Grundrüstzeug populationsdynamischer Analysen. Volterra, der schon in seiner Antrittsvorlesung für die Anwendung der Mathematik auf biologische Phänomene plädierte, wurde durch D'Ancona angeregt, sich mit den zwischen 1905 und 1923 in der Adria erhobenen Fischereistatistiken zu befassen; eine Beschäftigung, aus der die mathematische Modellierung einfacher Räuber-Beute-Verhältnisse auf der Grundlage von Differentialgleichungssystemen resultierte. Volterra interessierte sich für die sich anbahnende Synthese zwischen Darwinismus und Genetik; das Zukunftsträchtige dieser Kombination vor Augen hatte er nichts gegen die Zusammenarbeit mit den französischen Kollegen und Filmautoren, die seine Expertise beanspruchten, einzuwenden.

Als Volterra seinerseits Rat bei D'Ancona suchte, erwiderte dieser am 14. März 1937: «Ich schicke das Filmmanuskript über den Kampf ums Leben zurück. Ich las es und bin entsetzt. Wenn es die Absicht gewesen wäre, eine Parodie zu verfassen, wäre es akzeptabel, aber sicherlich nicht als etwas Seriöses.» Als Volterra seinen Willen zur Zusammenarbeit mit Kostitzin bekräftigte, doppelte D'Ancona am 18. März nach:

Ich glaube nicht, dass dieses Thema sich für Popularisierung eignet, ohne abgedroschen zu wirken. [...] Das ist es, was Kostitzin in meinen Augen getan hat; indem er Mendel, Mutationen und die Evolution von Pferden zusammenbringt, richtet er ein völliges Durch-

47 Volterra 1931. Im selben Jahr produzierte Painlevé auch einen weiteren Film mit dem Titel «Images mathématiques de la quatrième dimension».

48 Kostitzin legte im selben Jahr sein wichtiges Werk vor. Vgl. Kostitzin 1937.

49 Gasca 1996, 398. 
einander an. Ich halte es nicht für sinnvoll, dass sich ernsthafte wissenschaftliche Forscher an einer solchen grotesken Parodie beteiligen. ${ }^{50}$

Umgekehrt schrieb Kostitzin an Volterra von den enormen Schwierigkeiten, eine mathematische Theorie der natürlichen Selektion auf die Leinwand zu bringen. Am 5. April schrieb er nach Rom: «Das Skript ist sicher mangelhaft, denn es ist extrem schwierig, eine Anzahl von komplizierten Problemen im kurzen Zeitraum von 10 bis 12 Minuten zu illustrieren.» ${ }^{51}$ In der Folge korrespondierten die beiden immer wieder über Fragen der Mathematisierbarkeit der Biologie, wobei sie in der Wünschbarkeit eines solchen Unterfangens übereinstimmten.

Der Konflikt um die Beteiligung von Wissenschaftlern an einem populären Film war - im Unterschied zur Kontroverse um Einstein - nicht öffentlich. Auch Vito Volterra hatte die Repression des faschistischen Regimes direkt zu spüren bekommen; als er sich zu Beginn der 1930er Jahre weigerte, die vom Duce verlangten Loyalitätsbezeugungen zu leisten, wurde er aus allen Ämtern entlassen. Seine wissenschaftlichen Aktivitäten, die auch eine umfangreiche Korrespondenz mit Forschern aus Europa und Übersee umfassten, setzte er indessen fort. Das Engagement für eine Popularisierung wissenschaftlichen Wissens im Medium Film machte Divergenzen deutlich, die ansonsten wohl durch die pragmatischen Formen interdisziplinärer $\mathrm{Zu}$ sammenarbeit, wie sie sich zwischen Volterra und D'Ancona eingespielt hatte, überdeckt worden wären.

\section{Populäres Wissen und kinematographische Anschauung}

Wissenschaftliches Wissen lässt sich ohne Bildmedien weder gewinnen noch stabilisieren. Das sah Henri Bergson schon 1914, als er in der Berliner Zeitschrift «Der Kinematograph» die Analogien zwischen Film und Gedächtnis hervorhob und auf die «unendlichen Verdienste» und insbesondere auf «die grossen Vorteile der Kinematographie für die Naturwissenschaft» hinwies ${ }^{52}$. Analoge Überlegungen wurden auch von Regierungen gemacht. Beispielhaft ist die Stellungnahme des schweizerischen Bundesrates, der sich 1925 in einem Bericht zu einem sozialkonservativen parlamentarischen Postulat äusserte, das auf eine öffentliche Regulierung des «Kinematographengewerbes» abzielte. Die Landesregierung konzedierte zwar, «die gewaltige

50 Gasca 1996, 396f. [Übersetzung J.T.].

51 Gasca 1996, 397.

52 Henri Bergson, «Der Wert des Kinos», in: Der Kinematograph, Berlin, Nr. 379, 1. April 1914, zitiert aus: Schleif et al. 1989, 40. 
Entwicklung des Lichtspielwesens in den letzten Jahrzehnten und die fortschreitende Vervollkommnung der Technik» hätte «die Kinointeressenten dazu verleitet, ethisch minderwertige, auf die Sensationslust- und die niedern Instinkte der Massen berechnete dramatische Unterhaltungsstücke herzustellen und dem Publikum vorzuführen». Dies sei deswegen eine Gefahr, weil «viele dieser Filme [...] durch die Darstellung niedriger Leidenschaften und Begierden, grober Sinnlichkeit, sittlicher Laxheit und verwerflicher Handlungen verrohend und entsittlichend auf die Zuschauer ein[wirken]». Umgekehrt hob der Bericht die positiven Seiten des Films hervor. Es unterliege «keinem Zweifel, dass der Nutzbarmachung des Lichtspiels für Unterrichts- und wissenschaftliche Zwecke noch eine grosse Zukunft» bevorstehe: «Der Kinematograph kann weiten Volkskreisen als ein ausgezeichnetes Mittel der Belehrung dienen»; «dieser schönen Aufgabe» sei er aber «bis jetzt nicht oder jedenfalls nur in ungenügendem Masse gerecht geworden $»^{53}$.

Dieser Nutzen des Films für die Wissenschaft besteht nicht in der Anwendung des kinematographischen Mediums im experimentellen Forschungsprozess. Der Film über die «Images mathématiques» ist vielmehr ein Beispiel dafür, wie die «Botschaft», die durch «bewegte Bilder» einem breiten Publikum vermittelt werden soll, auf die innerwissenschaftliche Diskussion zurückwirkt und hier einen theoretisch klärenden Effekt haben kann. Die «Grosse Synthese» von Vererbungstheorie und Darwinismus, die das Thema dieses Films ist, ermöglichte es nämlich, das Verhältnis von Natur und Kultur neu zu denken und damit Distanz zu den sozialdarwinistischen Vermengungen von natürlichen Selektionsvorgängen und gesellschaftlichen Entwicklungen zu gewinnen, wie sie von Theorien des «Kampfes ums Dasein» propagiert wurden ${ }^{54}$. In dem, was in den Augen des Zoologen wie D'Ancona als «groteske Parodie» auf die Darwin'sche Lehre erscheinen musste, sah der Mathematiker Volterra, der aus seiner antifaschistischen Haltung nie einen Hehl gemacht hatte, einen neuen, rationalen Zugang zum Phänomen der natürlichen Selektion. Das Zusammenbringen von Mendel, Mutation und Pferdezucht öffnete ein Feld für populäre Demonstrationen komplexer Zusammenhänge. Die filmische Ästhetik bot die Möglichkeit für ein Spiel mit theoretischen Aussagen und visuellen Formen, das ein breiteres, an der Wissenschaft interessiertes Publikum faszinieren konnte. Gleichzeitig wirkten die Metaphern und Bilder, die der Film in populären Kontexten in Zirkulation versetzte und die dort «Sinn machten», wiederum zurück auf

53 Bericht des Bundesrates über das von Herrn Nationalrat Dr. Zimmerli und Mitunterzeichnern im Nationalrat eingereichte Postulat betr. Revision von Art. 31 der Bundesverfassung vom 26. Mai 1925, in: Schweizerisches Bundesblatt 1925/2, S. 545.

54 Siehe dazu Kronfeldner 2007. 
das Selbstverständnis der wissenschaftlichen Forschung, indem sie neue Hypothesen anregten, die Dokumentation der Laborarbeit verbesserten und - vermittelt durch die Entwicklung neuer Aufnahmetechniken - auch das Experimentieren selber beeinflussten.

Der Film über die Relativitätstheorie, der eineinhalb Jahrzehnte zuvor in einem filmtechnisch noch weit weniger entwickelten Umfeld hergestellt wurde, erreichte mit avancierten und avantgardistischen Gestaltungsprinzipien die visuelle Plausibilisierung einer kontraintuitiven, mit dem common sense nicht nachvollziehbaren wissenschaftlichen Theorie. Die «unnatürliche Natur der Wissenschaft» ${ }^{55}$ wurde mit trickreichen Darstellungsformen zugänglich gemacht; mit auf den Weltraum gerichteten Kanonen, die denen bei Jules Verne in nichts nachstanden, wurden physikalisch «unmögliche Situationen» genutzt, um eine mathematische Theorie der Physik mit Action zu unterfüttern. Und das sich rasch drehende Rad mit den Pistolen zeigt mittels eines die Aufmerksamkeit fesselnden Spektakels von Jahrmarktsqualität einen der wichtigen theoretischen Zusammenhänge auf. Das visuell sehr leistungsfähige und variationsreiche Medium Film fungiert in dieser Beziehung gleichsam als exoterische Einlösungsform einer esoterischen Wissenschaft ${ }^{56}$; indem es magische Momente in diese einfügt und sie in die sinnliche Präsenz zurückholt, stärkt es zugleich das Vertrauen in die Rationalität einer der menschlichen Sinneserfahrung nicht zugänglichen mathematisch fundierten Theorie ${ }^{57}$.

Auf der Leinwand wird die Welt zur lesbaren, zur dechiffrierbaren Oberfläche, wodurch sich auch die Beziehungen zwischen Menschen und Dingen verändern ${ }^{58}$. Die Freude am Formalisierbaren, die Lust, Apparate vorzuführen, positionieren den Menschen als ein Element innerhalb einer medial-materiellen Konstellation, die ihn dezentriert. Die «symbolische 〈Transposition〉 der Dinge und Körper in eine Wirklichkeit des Bildes» war eine Erfahrung, welche die Debatten um die Moderne der Zwischenkriegszeit massgeblich bestimmt hat ${ }^{59}$. Der populäre Wissenschaftsfilm ist ein aktives Moment dieser «Metamorphosen» - wie sie von Jacques Rancière genannt werden ${ }^{60}$. Sie treiben die Veränderung von Wahrnehmungs- und Erkenntnisweisen voran und ermöglichen neue Aussagen, ohne die Mehrdeutigkeit der bewegten Bilder zum Verschwinden zu bringen. Wissenschaft erliegt so immer wieder der Versuchung, ihre komplexen Verfahrensweisen

55 Wolpert 1992.

56 Nach einer Formulierung von Welsch 1997, 6.

57 Tröhler 2007.

58 Arburg et al. 2007.

59 Tröhler 2007, 291; vgl. auch $286 f$.

60 Rancière 2003, 43f. 
und ihre wenig nachvollziehbaren Resultate mittels eingängiger, ästhetisch attraktiver Visualisierungen zu plausibilisieren. Hirnforschung, Nanotechnologie und Astrophysik liefern laufend Beispiele dafür. Gegen die verallgemeinernde Ansicht, die neuen Bildmedien seien intrinsisch dem Okkulten verfallen ${ }^{61}$ und würden jene wissenschaftlichen Forschungsfelder, die sich ihrer bedienten, in ihren Bann schlagen, gilt es freilich zu sehen, dass der Einsatz von Bildern die kritische Selbstreflexivität wissenschaftlicher Praktiken auch erhöht und diese epistemisch sensibilisiert hat. Anstatt von einer die dunkle Seite der Aufklärung forcierenden Kolonialisierung wissenschaftlicher Forschung durch die Macht und Magie der Bilder zu sprechen, ist es sinnvoll, die Ambivalenz von Visualisierungsstrategien zu betonen, was impliziert, dass aus der Koppelung zwischen Wissenschaft und Mediensystem nach wie vor Chancen auch für die erstere resultieren.

\section{Bibliographie}

Achenbach, Michael/Paolo Caneppele/Ernst Kieninger, Projektionen der Sehnsucht: Saturn. Die erotischen Anfänge der österreichischen Kinematografie (Wien 2000)

Andriopoulos, Stefan, Besessene Körper. Hypnose, Körperschaft und die Erfindung des Kinos (München 2000)

Arburg, Hans G. et al. (Hrsg.), Mehr als Schein. Ästhetik der Oberfläche in Film, Kunst, Literatur und Theater (Berlin 2007)

Bawden, Liz-Anne (Hrsg.), Buchers Enzyklopädie des Films [deutsche Ausgabe hrsg. von Wolfram Tichy] (München/Luzern 1977)

Boussinot, Roger, L’Encyclopédie du cinéma (2 Bde.) (Paris 1995)

Crary, Jonathan, Techniken des Betrachters. Sehen und Moderne im 19. Jahrhundert (Dresden/ Basel 1996)

Daum, Andreas, Wissenschaftspopularisierung im 19. Jahrhundert: bürgerliche Kultur, naturwissenschaftliche Bildung und die deutsche Öffentlichkeit, 1848-1914 (München 2002)

Doering-Manteuffel, Sabine, Das Okkulte. Eine Erfolgsgeschichte im Schatten der Aufklärung. Von Gutenberg bis zum World Wide Web (München 2008)

Egli, Jeannette, Feind im Blut. Die Produktions-, Rezeptions- und Distributionsgeschichte eines frühen Syphilis-Films und die Aufklärungskampagne der Zürcherischen Gesellschaft zur Bekämpfung der Geschlechtskrankheiten in den Zwanziger- und Dreissigerjahren [unveröffentlichte Lizentiatsarbeit Universität Zürich] (Zürich 2000)

Eisenstaedt, Jean, The Curious History of Relativity. How Einstein's Theory of Gravity Was Lost and Found Again (Princeton N.J. 2006)

Elsaesser, Thomas, Metropolis. Der Filmklassiker von Fritz Lang (Hamburg 2000)

- Filmgeschichte und frühes Kino. Archäologie eines Medienwandels (München 2002)

Fleck, Ludwik, Entstehung und Entwicklung einer wissenschaftlichen Tatsache (Frankfurt a. M. 1980)

Franck, Georg, Ökonomie der Aufmerksamkeit: ein Entwurf (München 1998)

Frayling, Christopher, Mad, Bad and Dangerous. The Scientist and the Cinema (London 2002)

Gamper, Michael/Peter Schnyder (Hrsg.), Kollektive Gespenster. Die Masse, der Zeitgeist und andere unfassbare Körper (Freiburg i. Br. 2007)

61 Doering-Manteuffel 2008. 
Gasca, Ana Millán, "Mathematical theories versus biological facts: a debate on mathematical population dynamics in the 1930s", Historical Studies in the Physical and Biological Sciences 26 (1996) 347-403

Gunning, Tom, "The cinema of attractions: early film, its spectator and the avant-garde", in: Thomas Elsaesser/Adam Barker (eds), Early Cinema: Space, Frame, Narrative (London 1990) $56-62$

Hoffmann, Christoph, Unter Beobachtung. Naturforschung in der Zeit der Sinnesapparate (Göttingen 2006)

Hohmann, Joachim S., Sexualforschung und -aufklärung in der Weimarer Republik. Eine Übersicht in Materialien und Dokumenten; mit einem Beitrag über den frühen Aufklärungsfilm (Frankfurt a. M.) 1985

Kasten, Jürgen, «Wenn ein Mythos wieder Bild wird», Neue Zürcher Zeitung 158 (9. Juli 2008) 37

Kemner, Gerhard/Gelia Eisert, Lebende Bilder: eine Technikgeschichte des Films (Berlin 2000)

Kern, Stephen, The Culture of Time and Space 1880-1918 (London 1983)

Kostitzin, Vladimir A., Biologie mathématique (Paris 1937)

Krause, Marcus/Nicolas Pethes, «Einleitung: Die Kinematographie des Menschenversuchs», in: Marcus Krause/Nicolas Pethes (Hrsg.), Mr. Münsterberg und Dr. Hyde. Zur Filmgeschichte des Menschenexperiments (Bielefeld 2007) 7-15

Kronfeldner, Maria E., Darwinism, Memes, and Creativity: a critique of Darwinian analogical reasoning from nature to culture (o.O.2007) (Dissertationsmanuskript. Elektronische Datei: http://www.opus-bayern.de/uni-regensburg/volltexte/2007/801/pdf/Kronfeldner_Darwinism_ Memes_And_Creativity_opus.pdf)

Mocek, Reinhard, Biologie und soziale Befreiung. Zur Geschichte des Biologismus und der $<$ Rassenhygiene $>$ in der Arbeiterbewegung (Frankfurt a. M. et al. 2002)

Morat, Daniel, «Das Kino», in: Alexa Geisthövel (Hrsg.), Orte der Moderne. Erfahrungswelten des 19. und 20. Jahrhunderts (Frankfurt a. M. 2005) 228-237

Moritz, Verena/Karin Moser/Hannes Leidinger, Kampfzone Kino. Film in Österreich 1918-1938 (Wien 2008)

Müller, Corinna, «Der frühe Film, das frühe Kino und seine Gegner und Befürworter», in: Kaspar Maase (Hrsg.), Schund und Schönheit. Populäre Kultur um 1900 (Köln/Weimar/Wien 2001) 62-91

North, Douglass C., Structure and Change in Economic History (New York/London 1981)

Rancière, Jacques, Le destin des images (Paris 2003)

Renn, Jürgen (Hrsg.), Albert Einstein - Ingenieur des Universums. Dokumente eines Lebensweges (Berlin 2005)

Ross, Corey, "Mass culture and divided audiences: cinema and social change in Inter-War Germany", Past and Present 193 (2006) 157-196

Sarasin, Philipp, «Das obszöne Geniessen der Wissenschaft. Über Populärwissenschaft und $\mathrm{mad}$ scientists`», in: Philipp Sarasin (Hrsg.), Geschichtswissenschaft und Diskursanalyse (Frankfurt a. M. 2003) 231-257

Schirrmacher, Arne, «Nach der Popularisierung: zur Relation von Wissenschaft und Öffentlichkeit im 20. Jahrhundert», Geschichte und Gesellschaft 34 (2008) 73-95

Schleif, Helma et al. (Hrsg.), Stationen der Moderne im Film, Bd. 2 (Berlin 1989)

Schlüpmann, Heide, «Die 〈Optik des Lebens〉. Film als Triviales, Kino als Körperkunst», in: Kaspar Maase (Hrsg.), Schund und Schönheit. Populäre Kultur um 1900 (Köln/Weimar/Wien 2001) 92-105

Shepherd-Barr, Kirsten, Science on Stage. From Doctor Faustus to Copenhagen (Princeton N.J. 2006)

Skal, David, Screams of Reason. Mad Science and Modern Culture (New York/London 1998)

Tanner, Jakob, «Eugenik und Rassenhygiene in Wissenschaft und Politik seit dem ausgehenden 19. Jahrhundert: ein historischer Überblick», in: Michael Zimmermann (Hrsg.), Zwischen Erziehung und Vernichtung. Zigeunerpolitik und Zigeunerforschung im Europa des 20. Jahrhunderts (Stuttgart 2007) 109-121

Tröhler, Margrit, «Die sinnliche Präsenz der Dinge, oder: die skeptische Versöhnung mit der Moderne durch den Film», in: Christian Kiening (Hrsg.), Mediale Gegenwärtigkeit (Zürich 2007) 283-306 
Tudor, Andrew, Monsters and Mad Scientists. A Cultural History of the Horror Movie (Oxford 1989)

Turney, John, Frankenstein's Footsteps. Science, Genetics and Popular Culture (New Haven/ London 1998)

Verdicchio, Dirk Thomas, «Vom Aussen ins Innere (und wieder zurück). Medialisierung von Wissenschaft in Filmen über den Körper», Historische Anthropologie 16 (2008) 55-73

Verwoert, Jan, «Double Viewing:Versuch über die Bedeutung des 〈Pictorial Turn〉 für einen ideologiekritischen Umgang mit visuellen Medien - im Medium Videokunst», in: Jörg Huber (Hrsg.), Person/Schauplatz (Zürich/Wien/New York 2003) 223-241

Virilio, Paul, Krieg und Kino. Logistik der Wahrnehmung (München/Wien 1986)

Volterra, Vito, Leçons sur la théorie mathématique de la lutte pour la vie (Paris 1931)

von Keitz, Ursula, Im Schatten des Gesetzes. Schwangerschaftskonflikt und Reproduktion im deutschen Film 1918 bis 1933 (Marburg 2005)

- /Kay Hoffmann (Hrsg.), Die Einübung des dokumentarischen Blicks: «Fiction Film» und «Non Fiction Film» zwischen Wahrheitsanspruch und expressiver Sachlichkeit, 1895-1945 (Marburg 2001)

Wazeck, Milena, «Der verschollene Film. Interview von Thomas Bührke», Bild der Wissenschaft 2 (2005a) 47-49

- «Die verfilmte Realität», Spektrum der Wissenschaft 1 (2005b) 24-27

- «Einstein auf der Mordliste!〉 Die Angriffe auf Einstein und die Relativitätstheorie 1922», in: Jürgen Renn (Hrsg.), Albert Einstein - Ingenieur des Universums. Hundert Autoren für Einstein (Weinheim 2005c)

Weingart, Peter, Die Stunde der Wahrheit? Zum Verhältnis der Wissenschaft zu Politik, Wirtschaft und Medien in der Wissensgesellschaft (Weilerswist 2001)

- «Von Menschenzüchtern, Weltbeherrschern und skrupellosen Genies - das Bild der Wissenschaft im Spielfilm», in: Stefan Iglhaut (Hrsg.), Science + Fiction. Zwischen Nanowelt und globaler Kultur (Berlin 2003) 211-227

- Die Wissenschaft der Öffentlichkeit. Essays zum Verhältnis von Wissenschaft, Medien und Öffentlichkeit (Weilerswist 2005)

Welsch, Wolfgang, Unsere postmoderne Moderne (Berlin 1997)

Wolpert, Lewis, The Unnatural Nature of Science (London/Boston 1992) 